\title{
Towards a Vector Field Based Approach to the Proper Generalized Decomposition (PGD)
}

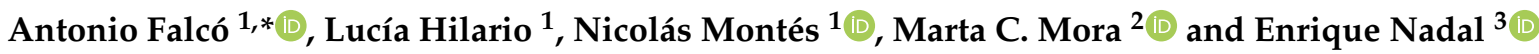 \\ 1 ESI International Chair@CEU-UCH, Departamento de Matemáticas, Física y Ciencias Tecnológicas, \\ Universidad Cardenal Herrera-CEU, CEU Universities San Bartolomé 55, 46115 Alfara del Patriarca, Spain; \\ luciah@uchceu.es (L.H.); nicolas.montes@uchceu.es (N.M.) \\ 2 Departamento de Ingeniería Mecánica y Construcción, Universitat Jaume I, Avd. Vicent Sos Baynat s/n, \\ 12071 Castellón, Spain; mmora@uji.es \\ 3 Departamento de Ingeniería Mecánica y de Materiales, Universitat Politècnica de València Camino de Vera, \\ s/n, 46022 Valencia, Spain; ennaso@upvnet.upv.es \\ * Correspondence: afalco@uchceu.es
}

Citation: Falcó, A.; Hilario, L.; Montés, N.; Marta, M.C.; Nadal, E. Towards a Vector Field Based Approach to the Proper Generalized Decomposition (PGD). Mathematics 2021, 9, 34. https: / /dx.doi.org/10.3390/math 9010034

\section{Received: 11 October 2020}

Accepted: 21 December 2020

Published: 25 December 2020

Publisher's Note: MDPI stays neutral with regard to jurisdictional claims in published maps and institutional affiliations.

Copyright: () 2020 by the authors. Licensee MDPI, Basel, Switzerland. This article is an open access article distributed under the terms and conditions of the Creative Commons Attribution (CC BY) license (https: / / creativecommons.org/ licenses/by/4.0/).

\begin{abstract}
A novel algorithm called the Proper Generalized Decomposition (PGD) is widely used by the engineering community to compute the solution of high dimensional problems. However, it is well-known that the bottleneck of its practical implementation focuses on the computation of the so-called best rank-one approximation. Motivated by this fact, we are going to discuss some of the geometrical aspects of the best rank-one approximation procedure. More precisely, our main result is to construct explicitly a vector field over a low-dimensional vector space and to prove that we can identify its stationary points with the critical points of the best rank-one optimization problem. To obtain this result, we endow the set of tensors with fixed rank-one with an explicit geometric structure.
\end{abstract}

Keywords: proper generalised decomposition; alternating least squares; greedy rank one update algorithm; tensor numerical methods

MSC: 15A69; 15A23; 57R25; 65N30

\section{Introduction}

Many problems in science and engineering are hard to compute due their numerical complexity. Moreover, in the analysis of complex systems under a real time constraint, the evaluation of all possible scenarios appears as a necessity [1]. Despite the improvements in techniques used in high dimensional problems, some challenging questions remain unresolved due to the efficiency of our computers. However, a novel technique called Proper Generalized Decomposition (PGD) [2,3] has been developed to provide an answer of these difficult tasks. It was initially proposed to compute, in a separated representation framework, the variational solution of partial differential equations (PDE) defined over a tensor product space [4]. It is possible to distinguish two different benefits. The first one is the possibility of managing high dimensional problems, and the second is the possibility to include the model's parameters as extra-coordinates. This last fact gives a powerful strategy to deal with classical problems because the PGD framework facilitates an efficient design and a real-time decision-making $[5,6]$.

This novel technique allows for computing the whole set of solutions of a parametrized problem. The strategy is to include in an equivalent non-parametrized problem all possible parameter values as extra-coordinates. The name of this particular PGD based approach is Progressive Variational Vademecum [1], and it can be implemented offline. As a consequence, the PGD based approach opens the possibility of solving problems in industry with a different strategy not envisioned until now. 
The mathematical analysis of the PGD was given by Falcó and Nouy, Ref. [4] in a Hilbert space framework and in [7] for a more general setting.

The Greedy Rank One Algorithm (GROA) [8] used to solve high-dimensional linear systems (with a full rank matrix) is the procedure of choice in the engineering community to implement the PGD. It is an iterative method made up of two steps that we can cyclically repeat until convergence. The first one consists of computing the minimal residual of the linear system over the set of tensors with bounded rank-one. In the second step, we use this optimal rank-one solution to update the residual. In the following, we return to the first step. Ammar et al. [8] propose an Alternating Least Squares (ALS) Algorithm for the practical implementation of the first step of the GROA. In the aforementioned paper, the authors justify the choice of the ALS showing that its convergence to a critical point of the optimization problem (not necessarily an optimal one) is assured under very weak conditions. In addition, the convergence has been studied by El Hamidi, Osman and Jazar [9] in the framework of Sobolev tensor spaces.

In this work, we want to study the optimization problem of the first step the GROA. To this end, even though our problem is not convex, we will take into account the relationship between the underlying convex optimization problem and the behaviour of its associated gradient flow. It is well-known that the vector field constructed by using a convex functional (for example related with a convex minimization problem defined over a finite dimensional vector space), has a gradient flow that provides a dynamical system with a unique stationary point. Moreover, it can be shown that it is a sink and its stable manifold coincides with the whole domain of the convex functional. This fact motivates the classical paradigm about the convergence of gradient-based numerical optimization algorithms.

In this work, we want to adapt the above paradigm in the framework of the GROA [8]. The main goal is to find a vector field in a low-dimensional vector space related to the gradient flow of a convex functional defined over the set of tensors of fixed rank one. The idea is to use this vector field to characterize the behaviour of the solutions of the non-convex optimization problem associated with the first step of GROA. To achieve this, we will prove that the set of critical points of the optimization problem over the set of tensors with fixed rank-one can be identified with the set of stationary points of that vector field. In order to construct it, we will proceed as follows. First, we will endow the set tensors of fixed rank-one with an explicit structure of smooth manifold. Second, by the help of this geometric structure, we will explicitly construct a vector field over a low dimensional vector space related with the first step of GROA. Finally, we will show that the set of stationary points of this vector field coincides with the set of critical points of the optimization problem associated with the PGD algorithm. In consequence, this vector field allows us to explain the dynamical behaviour around each of its stationary points. Moreover, we can get explicit information, in a neighbourhood of each of these stationary points, about the structure of its stable and unstable manifolds. In our opinion, a more precise knowledge of these invariant sets can help us develop better and more efficient PGD approaches.

The paper is organised as follows. Section 2 provides some preliminary definitions and results used along this paper. Section 3 shows a geometric approach to the PGD. In Section 4, the characterization of the smooth manifold of the set of tensors of fixed rank-one is given. After that, Section 5 shows the first order optimality conditions for the PGD which is the main result of this paper. Finally, Section 6 provides some conclusions of the work.

\section{Preliminary Definitions and Results}

First of all, we introduce some notation used along this paper. We denote by $\mathbb{R}^{N \times M}$ the set of $N \times M$-matrices and by $A^{T}$ the transpose of a given matrix $A$. As usual, we use

$$
\langle\mathbf{x}, \mathbf{y}\rangle=\mathbf{x}^{T} \mathbf{y}=\mathbf{y}^{T} \mathbf{x}
$$


to denote the Euclidean inner product in $\mathbb{R}^{N}$, and its corresponding 2-norm, by $\|x\|_{2}=$ $\langle\mathbf{x}, \mathbf{x}\rangle^{1 / 2}$. Let $I_{N}$ be the $N \times N$-identity matrix and when the dimension is clear from the context, we simply denote it by $I$. Given a sequence $\left\{\mathbf{u}_{j}\right\}_{j=0}^{\infty} \subset \mathbb{R}^{N}$, we say that a vector $\mathbf{u} \in \mathbb{R}^{N}$ can be written as

$$
\mathbf{u}=\sum_{j=0}^{\infty} \mathbf{u}_{j}
$$

if and only if

$$
\lim _{n \rightarrow \infty} \sum_{j=0}^{n} \mathbf{u}_{j}=\mathbf{u}
$$

in the $\|\cdot\|_{2}$-topology. Now, we recall the definition and some properties of the Kronecker product. The Kronecker product of $A \in \mathbb{R}^{N_{1}^{\prime} \times N_{1}}$ and $B \in \mathbb{R}^{N_{2}^{\prime} \times N_{2}}$, written $A \otimes B$, is the tensor algebraic operation defined as

$$
A \otimes B=\left[\begin{array}{cccc}
A_{1,1} B & A_{1,2} B & \cdots & A_{1, N_{1}^{\prime}} B \\
A_{2,1} B & A_{2,2} B & \cdots & A_{2, N_{1}^{\prime}} B \\
\vdots & \vdots & \ddots & \vdots \\
A_{N_{1}, 1} B & A_{N_{1}, 2} B & \cdots & A_{N_{1}, N_{1}^{\prime}} B
\end{array}\right] \in \mathbb{R}^{N_{1}^{\prime} N_{2}^{\prime} \times N_{1} N_{2}} .
$$

To conclude, we list some of the well-known properties of the Kronecker product (see, for example, [10] or [11]).

1. $A \otimes(B \otimes C)=(A \otimes B) \otimes C$.

2. $(A+B) \otimes(C+D)=(A \otimes C)+(B \otimes C)+(A \otimes D)+(B \otimes D)$.

3. $A B \otimes C D=(A \otimes C)(B \otimes D)$.

4. $(A \otimes B)^{-1}=A^{-1} \otimes B^{-1}$.

5. $(A \otimes B)^{T}=A^{T} \otimes B^{T}$.

6. If $A$ and $B$ are banded, then $A \otimes B$ is banded.

7. If $A$ and $B$ are symmetric, then $A \otimes B$ is symmetric.

8. If $A$ and $B$ are definite positive, then $A \otimes B$ is definite positive.

The concept of separated representation was introduced by Beylkin and Mohlenkamp in [12], and it is related to the problem of constructing the approximate solutions of some classes of problems in high-dimensional spaces by means of a separable function. In particular, for a given map

$$
u:[0,1]^{d} \subset \mathbb{R}^{d} \longrightarrow \mathbb{R}
$$

we say that it has a separable representation if

$$
u\left(x_{1}, \ldots, x_{d}\right)=\sum_{j=1}^{\infty} u_{1}^{(j)}\left(x_{1}\right) \cdots u_{d}^{(j)}\left(x_{d}\right)
$$

Now, consider a mesh of $[0,1]$ in the $x_{k}$-variable given by $N_{k}$-mesh points, $1 \leq k \leq d$, then we can write a discrete version of (1) by

$$
u\left(x_{i_{1}}, \ldots, x_{i_{d}}\right)=\sum_{j=1}^{\infty} u_{1}^{(j)}\left(x_{i_{1}}\right) \cdots u_{d}^{(j)}\left(x_{i_{d}}\right)
$$

where $1 \leq i_{k} \leq N_{k}$ for $1 \leq k \leq d$. Observe that, for each $1 \leq k \leq d$, if $\mathbf{x}_{k}^{j} \in \mathbb{R}^{N_{k}}$ denotes the vector with components $u_{k}^{(j)}\left(x_{i_{k}}\right)$ for $1 \leq i_{k} \leq N_{k}$, then (2) is equivalent to

$$
\mathbf{u}=\sum_{j=1}^{\infty} \mathbf{x}_{1}^{j} \otimes \cdots \otimes \mathbf{x}_{d}^{j}
$$


We point out that (3) is a useful expression to implement numerical algorithms using the MATLAB and OCTAVE function kron.

Suppose that, for given a linear Partial Differential Equation, and after a discretization by means of Finite Elements, we need to solve the linear system:

$$
A \mathbf{u}=\mathbf{f}
$$

where $A$ is a $\left(N_{1} \cdots N_{d}\right) \times\left(N_{1} \cdots N_{d}\right)$-dimensional invertible matrix, for some $N_{1}, \ldots, N_{d} \in$ $\mathbb{N}$, that is, $A \in \mathrm{GL}\left(N_{1} \cdots N_{d}\right)$. Then, from all said above, a low rank approximation

$$
A^{-1} \mathbf{f} \approx \mathbf{u}_{n}=\sum_{j=1}^{n} \mathbf{x}_{1}^{j} \otimes \cdots \otimes \mathbf{x}_{d}^{j}
$$

with sufficient approximation exists, for some $n \geq 1$ and where $\mathbf{x}_{i}^{j} \in \mathbb{R}^{N_{i}}$ for $i=$ $1,2, \ldots, d$ and $j=1,2, \ldots, n$. Moreover, we would show that

$$
\lim _{n \rightarrow \infty}\left\|A^{-1} \mathbf{f}-\mathbf{u}_{n}\right\|_{2}=0
$$

that is,

$$
A^{-1} \mathbf{f}=\sum_{j=1}^{\infty} \mathbf{x}_{1}^{j} \otimes \cdots \otimes \mathbf{x}_{d}^{j}
$$

Thus, in a first approach to solve it, we would like to determine vectors $\mathbf{x}_{1}^{j}, \ldots, \mathbf{x}_{d}^{j}$ for $j=1,2, \ldots, n$ that minimizes

$$
\left\|\mathbf{f}-A\left(\sum_{j=1}^{n} \mathbf{x}_{1}^{j} \otimes \cdots \otimes \mathbf{x}_{d}^{j}\right)\right\|_{2},
$$

or, in short

$$
\operatorname{argmin}_{\operatorname{rank}_{\otimes} \mathbf{u} \leq n}\|\mathbf{f}-A \mathbf{u}\|_{2},
$$

by using the notation introduced in [13].

The Proper Generalised Decomposition (PGD in short) appears when we consider solving the linear Equation (4) as an optimization problem as follows. For each fixed $A \in \mathrm{GL}\left(N_{1} N_{2} \cdots N_{d}\right)$ and $\mathbf{f} \in \mathbb{R}^{N_{1} N_{2} \cdots N_{d}}$, we define a map

$$
J_{A, \mathbf{f}}: \mathbb{R}^{N_{1} \cdots N_{d}} \rightarrow \mathbb{R}, \quad J_{A, \mathbf{f}}(\mathbf{u})=\frac{1}{2}\|\mathbf{f}-A \mathbf{u}\|_{2}^{2},
$$

hence

$$
\operatorname{argmin}_{\mathbf{u} \in \mathbb{R}^{N_{1} N_{2} \cdots N_{d}}} J_{A, \mathbf{f}}(\mathbf{u})=\left\{A^{-1} \mathbf{f}\right\}
$$

holds. The goal is to use (5) to approximate the solution of (4). To this end, for each $n \in \mathbb{N}$, we define the set

$$
\mathcal{S}_{n}=\left\{\mathbf{x} \in \mathbb{R}^{N_{1} \cdots N_{d}}: \operatorname{rank}_{\otimes} \mathbf{x} \leq n\right\},
$$

introduced in [13], in the following way. Given $\mathbf{x} \in \mathbb{R}^{N_{1} \cdots N_{d}}$, we say that $\mathbf{x} \in \mathcal{S}_{1}=$ $\mathcal{S}_{1}\left(N_{1}, N_{2}, \ldots, N_{d}\right)$ if $\mathbf{x}=\mathbf{x}_{1} \otimes \mathbf{x}_{2} \otimes \cdots \otimes \mathbf{x}_{d}$, where $\mathbf{x}_{i} \in \mathbb{R}^{N_{i}}$, for $i=1, \ldots, d$. For $n \geq 2$, we define inductively $\mathcal{S}_{n}=\mathcal{S}_{n}\left(N_{1}, N_{2}, \ldots, N_{d}\right)=\mathcal{S}_{n-1}+\mathcal{S}_{1}$, that is,

$$
\mathcal{S}_{n}=\left\{\mathbf{x}: \mathbf{x}=\sum_{i=1}^{k} \mathbf{x}^{(i)}, \mathbf{x}^{(i)} \in \mathcal{S}_{1} \text { for } 1 \leq i \leq k \leq n\right\} .
$$

Note that $\mathcal{S}_{n} \subset \mathcal{S}_{n+1}$ for all $n \geq 1$. 
Unfortunately, from Proposition 4.1 (a) of [13], we have that the set $\mathcal{S}_{n}$ is not necessarily (or even usually) closed for each $n \geq 2$. However, from Proposition 4.2 of [13], it follows that $\mathcal{S}_{1}$ is a closed set in any norm-topology. This fact implies (see Lemma 1 in [8]), that given $A \in \mathrm{GL}\left(N_{1} N_{2} \cdots N_{d}\right)$, then, for every $\mathbf{f} \in \mathbb{R}^{N_{1} \cdots N_{d}}$, we have that the set

$$
\mathfrak{C}(A, \mathbf{f}):=\operatorname{argmin}_{\mathbf{x} \in \mathcal{S}_{1}} J_{A, \mathbf{f}}(\mathbf{x}) \neq \varnothing .
$$

This allows for considering the following iterative scheme. Let $\mathbf{u}_{0}=\mathbf{y}_{0}=\mathbf{0}$, and, for each $n \geq 1$, take

$$
\begin{aligned}
\mathbf{r}_{n-1} & =\mathbf{f}-A \mathbf{u}_{n-1} \\
\mathbf{u}_{n} & =\mathbf{u}_{n-1}+\mathbf{y}_{n} \text { where } \mathbf{y}_{n} \in \mathfrak{C}\left(A, \mathbf{r}_{n-1}\right)
\end{aligned}
$$

Note that, given $A \in \operatorname{GL}\left(N_{1} N_{2} \cdots N_{d}\right)$ and $\mathbf{f} \in \mathbb{R}^{N_{1} \cdots N_{d}}$, we can construct for each $n$, by using (8) and (9), a vector

$$
\mathbf{u}_{n}=\sum_{j=1}^{n} \mathbf{y}_{n} \in \mathcal{S}_{n} \backslash \mathcal{S}_{n-1}
$$

Here, we assume that $\mathbf{y}_{j} \neq \mathbf{0}$ for $1 \leq j \leq n$, that is, $\operatorname{rank}_{\otimes} \mathbf{u}_{n}=n$. Since $\mathbf{u}_{n} \approx A^{-1} \mathbf{f}$, we define the $\operatorname{rank}_{\otimes}$ for $A^{-1} \mathbf{f}$ obtained by the Greedy Rank-One Update Algorithms (8) and (9) as

$$
\operatorname{rank}_{\otimes}^{G}\left(A^{-1} \mathbf{f}\right)=\left\{\begin{array}{cc}
\infty & \text { if }
\end{array} \begin{array}{c}
\left\{j \geq 1: \mathbf{y}_{j}=\mathbf{0}\right\}=\varnothing, \\
\text { otherwise. }
\end{array}\right.
$$

The following theorem (see Theorem 1 in [8]) gives the convergence of the Greedy Rank-One Update Approximation for solving linear systems with full rank matrix.

Theorem 1. Let $\mathbf{f} \in \mathbb{R}^{N_{1} N_{2} \cdots N_{d}}$ and $A \in \mathrm{GL}\left(N_{1} N_{2} \cdots N_{d}\right)$. Then, by using the iterative scheme (8) and (9), we obtain that the sequence $\left\{\left\|\mathbf{r}_{n}\right\|_{2}\right\}_{n=0}^{\operatorname{rank}_{\otimes}^{G}\left(A^{-1} \mathbf{f}\right)}$, is strictly decreasing and

$$
A^{-1} \mathbf{f}=\lim _{n \rightarrow \infty} \mathbf{u}_{n}=\sum_{j=0}^{\operatorname{rank}_{\otimes}^{G}\left(A^{-1} \mathbf{f}\right)} \mathbf{y}_{j}
$$

Moreover, the rate of convergence is given by

$$
\frac{\left\|\mathbf{r}_{n}\right\|_{2}}{\left\|\mathbf{r}_{0}\right\|_{2}}=\prod_{j=1}^{n} \sin \theta_{j}
$$

for $1 \leq n \leq \operatorname{rank}_{\otimes}^{G}\left(A^{-1} \mathbf{f}\right)$ where

$$
\theta_{j}=\arccos \left(\frac{\left\langle\mathbf{r}_{j-1}, A \mathbf{y}_{j}\right\rangle}{\left\|\mathbf{r}_{j-1}\right\|_{2}\left\|A \mathbf{y}_{j}\right\|_{2}}\right) \in(0, \pi / 2)
$$

for $1 \leq j \leq n$.

From (10), we obtain that, if $\operatorname{rank}_{\otimes}^{G}\left(A^{-1} \mathbf{f}\right)<\infty$, then $\left\|\mathbf{r}_{n}\right\|_{2}=0$ for all $n>\operatorname{rank}_{\otimes}^{G}\left(A^{-1} \mathbf{f}\right)$. Thus, the above theorem allows for us to construct a procedure, which we give in the pseudo-code form in Algorithm 1, under the assumption that we have a numerical method in order to find a y solving (7) (see the step 5 in Algorithm 1) and that we introduce below. 


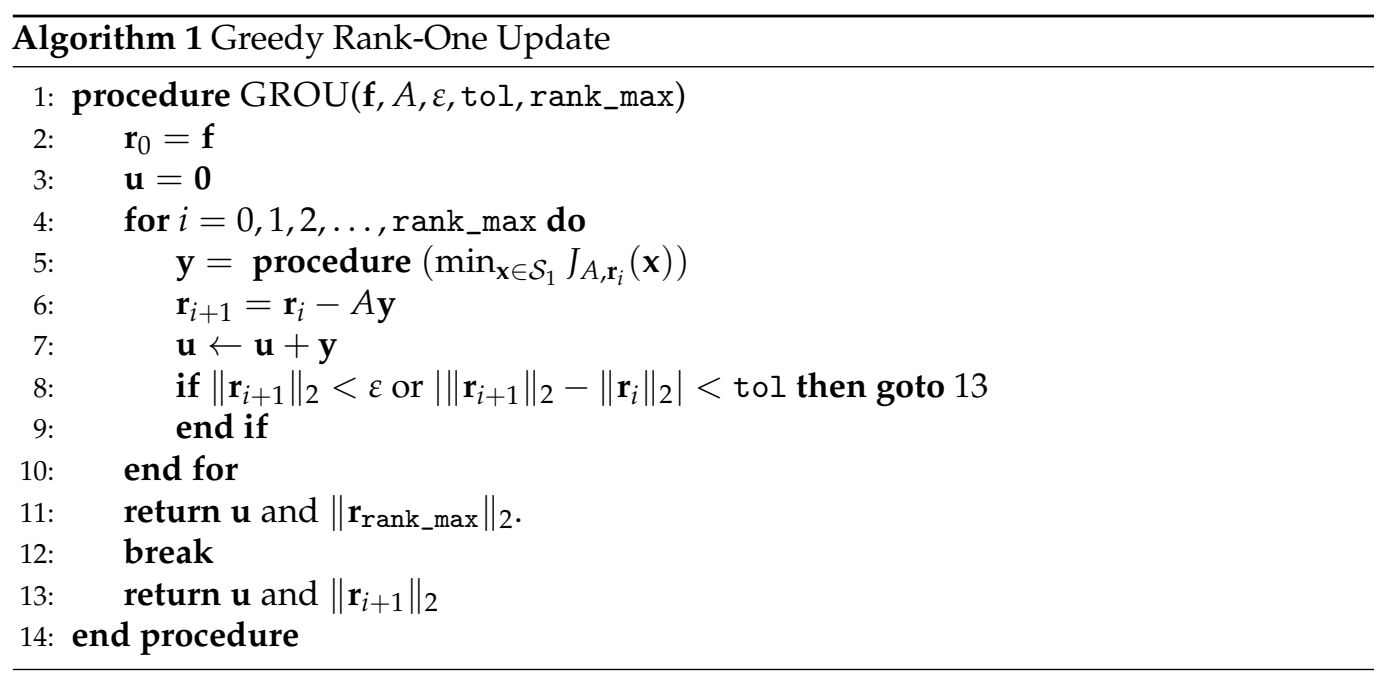

\section{A Geometric Approach to the PGD}

In this section, to study the procedure given in the line 5 of the Algorithm 1, we introduce a smooth manifold. To this end, introduce first the set of tensors of fixed rank-one in the tensor space $\mathbb{R}^{N_{1} \cdots N_{d}}=\bigotimes_{j=1}^{d} \mathbb{R}^{N_{j}}$ defined as

$$
\mathcal{M}_{N_{1} \cdots N_{d}}=\left\{\mathbf{u} \in \bigotimes_{j=1}^{d} \mathbb{R}^{N_{j}}: \mathbf{u}=\lambda \bigotimes_{j=1}^{d} \mathbf{u}_{j}, \lambda \in \mathbb{R}_{*}, \mathbf{u}_{j} \in \mathbb{R}^{N_{j}} \backslash\{\mathbf{0}\}, 1 \leq j \leq d\right\}
$$

where $\mathbb{R}_{*}=\mathbb{R} \backslash\{0\}$. Observe that the set

$$
\mathcal{S}_{1}=\{\mathbf{0}\} \cup \mathcal{M}_{N_{1} \cdots N_{d}} .
$$

Then, our first result is the following theorem of the alternative.

Theorem 2. Let $A \in \mathrm{GL}\left(N_{1} N_{2} \cdots N_{d}\right)$ and $\mathbf{f} \in \mathbb{R}^{N_{1} N_{2} \cdots N_{d}}$. Either $\mathfrak{C}(A, \mathbf{f}) \subset \mathcal{M}_{N_{1} \cdots N_{d}}$, or $\mathbf{0} \in$ $\mathfrak{C}(A, \mathbf{f})$ but not both.

Proof. Assume that $\mathbf{0} \in \mathfrak{C}(A, \mathbf{f})$ and that there exists $\mathbf{u} \in \mathfrak{C}(A, \mathbf{f}) \cap \mathcal{M}_{N_{1} \cdots N_{d}}$. Since we can write

$$
J_{A, \mathbf{f}}(\mathbf{u})=\frac{1}{2}\|\mathbf{f}\|_{2}^{2}-\mathbf{f}^{T} A \mathbf{u}+\frac{1}{2}\|A \mathbf{u}\|_{2}^{2}
$$

and $J_{A, \mathbf{f}}(\mathbf{0})=J_{A, \mathbf{f}}(\mathbf{u})=\frac{1}{2}\|\mathbf{f}\|_{2}^{2} \leq J_{A, \mathbf{f}}\left(\mathbf{u}^{\prime}\right)$ holds for all $\mathbf{u}^{\prime} \in \mathcal{M}_{N_{1} \cdots N_{d^{\prime}}}$ we have

$$
\mathbf{f}^{T} A \mathbf{u}=\frac{1}{2}\|A \mathbf{u}\|_{2}^{2}>0 .
$$

Now, consider the map $f: \mathbb{R} \longrightarrow \mathbb{R}$ defined as

$$
f(\lambda):=J_{A, \mathbf{f}}(\lambda \mathbf{u})=\frac{1}{2}\|\mathbf{f}\|_{2}^{2}-\lambda \mathbf{f}^{T} A \mathbf{u}+\frac{\lambda^{2}}{2}\|A \mathbf{u}\|_{2}^{2}=\frac{1}{2}\|\mathbf{f}\|_{2}^{2}-\mathbf{f}^{T} A \mathbf{u} \lambda+\mathbf{f}^{T} A \mathbf{u} \lambda^{2} .
$$

Then, $f(0)=J_{A, \mathbf{f}}(\mathbf{0})=J_{A, \mathbf{f}}(\mathbf{u})=f(1) \leq f(\lambda)$ for all $\lambda \in \mathbb{R}$ holds. Observe that

$$
f^{\prime}(\lambda)=-\mathbf{f}^{T} A \mathbf{u}+2 \mathbf{f}^{T} A \mathbf{u} \lambda
$$

and hence the map $f$ has a global minimum for $\lambda=\frac{1}{2}$, a contradiction. 
The main consequence of this result is the following. It says that the output of the procedure given in step 5 in Algorithm 1 always remains in the set $\mathcal{M}_{N_{1} \cdots N_{d}}$ before it gives us the final output.

Corollary 1. Let $\mathbf{f} \in \mathbb{R}^{N_{1} N_{2} \cdots N_{d}}$ and $A \in \mathrm{GL}\left(N_{1} N_{2} \cdots N_{d}\right)$ such that $A^{-1} \mathbf{f} \notin \mathcal{S}_{1}$. Then, $\operatorname{rank}_{\otimes}^{G}\left(A^{-1}\right)=i-1$ for some $i>1$ if and only if $\mathfrak{C}\left(A, \mathbf{r}_{i}\right)=\{\mathbf{0}\}$, and hence $\mathfrak{C}\left(A, \mathbf{r}_{j}\right) \subset$ $\mathcal{M}_{N_{1} \cdots N_{d}}$ for all $j<i-1$.

As a consequence of the above corollary, the situation of interest is when given $A \in \mathrm{GL}\left(N_{1} N_{2} \cdots N_{d}\right)$ and $\mathbf{f} \in \mathbb{R}^{N_{1} N_{2} \cdots N_{d}}$, we have that $\mathfrak{C}(A, \mathbf{f}) \subset \mathcal{M}_{N_{1} \cdots N_{d}}$. Thus, in order to study the vectors in $\mathfrak{C}(A, \mathbf{f})$, we need to characterize the structure of the critical points of the map $\mathbf{u} \mapsto\|\mathbf{f}-A \mathbf{u}\|$ restricted to the set $\mathcal{M}_{N_{1} \cdots N_{d}}$. To see this in the next section, we provide to $\mathcal{M}_{N_{1} \cdots N_{d}}$ of a structure of smooth manifold.

\section{The Set of Tensors of Fixed Rank-One as a Smooth Manifold}

Along this paper, we will consider a manifold as a pair $(\mathbb{M}, \mathcal{A})$, where $\mathbb{M}$ is a subset of some finite-dimensional vector space $V$ and $\mathcal{A}$ is an atlas representing the local coordinate system of $\mathbb{M}$. We recall the definition of an atlas associated with a set $\mathbb{M}$.

Definition 1. Let $\mathbb{M}$ be a set. An atlas of class $C^{p}(p \geq 0)$ or analytic on $\mathbb{M}$ is a family of charts with some indexing set $A$, namely $\left\{\left(U_{\alpha}, \varphi_{\alpha}\right): \alpha \in A\right\}$, having the following properties (see [14]):

AT1 $\left\{U_{\alpha}\right\}_{\alpha \in A}$ is a covering of $\mathbb{M}$, that is, $U_{\alpha} \subset \mathbb{M}$ for all $\alpha \in A$ and $\cup_{\alpha \in A} U_{\alpha}=\mathbb{M}$.

AT2 For each $\alpha \in A,\left(U_{\alpha}, \varphi_{\alpha}\right)$ stands for a bijection $\varphi_{\alpha}: U_{\alpha} \rightarrow W_{\alpha}$ of $U_{\alpha}$ onto an open set $W_{\alpha}$ of a finite dimensional normed space $\left(X_{\alpha},\|\cdot\|_{\alpha}\right)$, and for any $\alpha$ and $\beta$ the set $\varphi_{\alpha}\left(U_{\alpha} \cap U_{\beta}\right)$ is open in $X_{\alpha}$.

AT3 Finally, if we let $U_{\alpha} \cap U_{\beta}=U_{\alpha, \beta}$ and $\varphi_{\alpha}\left(U_{\alpha, \beta}\right)=U_{\alpha, \beta}$, the transition mapping $\varphi_{\beta} \circ \varphi_{\alpha}^{-1}$ : $U_{\alpha, \beta} \rightarrow U_{\beta, \alpha}$ is a diffeomorphism of class $\mathcal{C}^{p}(p \geq 0)$ or analytic.

Observe that the condition of an open covering is not used, see [14]. Moreover, in AT2, we do not require that the normed spaces to be the same for all indices $\alpha$, or even to be isomorphic. If $X_{\alpha}$ is linearly isomorphic to some finite dimensional normed space $X$ for all $\alpha$, we have the following definition.

Definition 2. Let $\mathbb{M}$ be a set and $X$ be a finite dimensional normed space. We say that $\mathbb{M}$ is a $C^{p}$ (respectively, analytic) manifold modelled on $X$ if there exists an atlas of class $C^{p}$ (respectively, analytic) over $\mathbb{M}$ with $X_{\alpha}$ linearly isomorphic to $X$ for all $\alpha \in A$.

Since different atlases can give the same manifold, we say that two atlases are compatible if each chart of one atlas is compatible with the charts of the other atlas in the sense of AT3. One verifies that the relation of compatibility between atlases is an equivalence relation.

Definition 3. An equivalence class of atlases of class $C^{p}$ on $\mathbb{M}$, also denoted by $\mathcal{A}$, is said to define a structure of a $C^{p}$-manifold on $\mathbb{M}$, and hence we say that $(\mathbb{M}, \mathcal{A})$ is a finite dimensional manifold. In a similar way, if an equivalence class of atlases is given by analytic maps, then we say that $(\mathbb{M}, \mathcal{A})$ is an analytic finite dimensional manifold.

For each $\mathbf{u}=\lambda \bigotimes_{j=1}^{d} \mathbf{u}_{j} \in \mathcal{M}_{N_{1} \cdots N_{d}}$, we construct a local chart as follows. Let be

$$
\operatorname{span}\left\{\mathbf{u}_{j}\right\}^{\perp}=\left\{\mathbf{v}_{j} \in \mathbb{R}^{N_{j}}: \mathbf{v}_{j}^{T} \cdot \mathbf{u}_{j}=0\right\},
$$

the orthogonal complement of the linear space $\operatorname{span}\left\{\mathbf{u}_{j}\right\}$ for $1 \leq j \leq d$. Let us consider the set

$$
\mathcal{U}_{\mathbf{u}}:=\operatorname{span}\left\{\mathbf{u}_{1}\right\}^{\perp} \times \cdots \times \operatorname{span}\left\{\mathbf{u}_{d}\right\}^{\perp} \times \mathbb{R}_{*},
$$


which is an open and dense set of the finite-dimensional vector space

$$
X_{\mathbf{u}}:=\operatorname{span}\left\{\mathbf{u}_{1}\right\}^{\perp} \times \cdots \times \operatorname{span}\left\{\mathbf{u}_{d}\right\}^{\perp} \times \mathbb{R} .
$$

Observe that the vector space $X_{\mathbf{u}}$ is linearly isomorphic to the vector space $\mathbb{R}^{N_{1}-1} \times$ $\cdots \times \mathbb{R}^{N_{d}-1} \times \mathbb{R}$ for all $\mathbf{u} \in \mathcal{M}_{N_{1} \cdots N_{d}}$.

Now, we introduce the set

$$
\mathfrak{U}(\mathbf{u}):=\left\{\begin{array}{ll}
\mathbf{u}^{\prime} \in \mathcal{M}_{N_{1} \cdots N_{d}}: \mathbf{u}^{\prime}=\beta \bigotimes_{j=1}^{d}\left(\mathbf{u}_{j}+\mathbf{w}_{j}\right), \begin{array}{l}
\mathbf{w}_{j} \in \operatorname{span}\left\{\mathbf{u}_{j}\right\}^{\perp}, 1 \leq j \leq d \\
\beta \in \mathbb{R}_{*}
\end{array}
\end{array}\right\}
$$

in $\mathcal{M}_{N_{1} \cdots N_{d}}$ for which we can construct a natural bijection:

$$
\varphi_{\mathbf{u}}: \mathfrak{U}(\mathbf{u}) \longrightarrow \mathcal{U}_{\mathbf{u}}, \quad \mathbf{u}^{\prime} \mapsto\left(\mathbf{w}_{1}, \ldots, \mathbf{w}_{d}, \beta\right) \Leftrightarrow \mathbf{u}^{\prime}=\beta \bigotimes_{j=1}^{d}\left(\mathbf{u}_{j}+\mathbf{w}_{j}\right) .
$$

Then, we can state the following result.

Theorem 3. The set $\mathcal{A}_{N_{1} \cdots N_{d}}=\left\{\left(\mathfrak{U}(\mathbf{u}), \varphi_{\mathbf{u}}\right): \mathbf{u} \in \mathcal{M}_{N_{1} \cdots N_{d}}\right\}$ is an atlas for $\mathcal{M}_{N_{1} \cdots N_{d}}$ and hence $\left(\mathcal{M}_{N_{1} \cdots N_{d}}, \mathcal{A}_{N_{1} \cdots N_{d}}\right)$ is a $\mathcal{C}^{\infty}$-manifold modelled on

$$
\mathbb{R}^{N_{1}-1} \times \cdots \times \mathbb{R}^{N_{d}-1} \times \mathbb{R} \cong \mathbb{R}^{\log _{2}\left(2^{\left.N_{1} \cdots N_{d}\right)-d+1} .\right.}
$$

Proof. Clearly, AT1 holds. To prove AT2 and AT3, let us consider $\mathbf{u}, \mathbf{u}^{\prime} \in \mathcal{M}_{N_{1} \cdots N_{d}}$ be such that $\mathfrak{U}(\mathbf{u}) \cap \mathfrak{U}\left(\mathbf{u}^{\prime}\right) \neq \varnothing$. Without loss of generality we may assume that $\mathbf{u}=\lambda \otimes_{j=1}^{d} \mathbf{u}_{i}$ and $\mathbf{u}^{\prime}=\lambda^{\prime} \bigotimes_{j=1}^{d} \mathbf{u}_{i^{\prime}}^{\prime}$, where $\left\|\mathbf{u}_{i}\right\|_{i, 2}=\left\|\mathbf{u}_{i}^{\prime}\right\|_{i, 2}=1$ for $1 \leq i \leq d$. Then, for each $\mathbf{z} \in \mathfrak{U}(\mathbf{u}) \cap \mathfrak{U}\left(\mathbf{u}^{\prime}\right)$, there exists a unique $\left(\mathbf{w}_{1}, \ldots, \mathbf{w}_{d}, \beta\right) \in \mathcal{U}_{\mathbf{u}}$ and a unique $\left(\mathbf{w}_{1}^{\prime}, \ldots, \mathbf{w}_{d^{\prime}}^{\prime}, \beta^{\prime}\right) \in \mathcal{U}_{\mathbf{u}^{\prime}}$ such that

$$
\mathbf{z}=\beta \bigotimes_{j=1}^{d}\left(\mathbf{u}_{j}+\mathbf{w}_{j}\right)=\beta^{\prime} \bigotimes_{j=1}^{d}\left(\mathbf{u}_{j}^{\prime}+\mathbf{w}_{j}^{\prime}\right)
$$

Since

$$
\operatorname{span}\left\{\mathbf{u}_{j}+\mathbf{w}_{j}\right\}=\operatorname{span}\left\{\mathbf{u}_{j}^{\prime}+\mathbf{w}_{j}^{\prime}\right\}
$$

holds, there exists a unique $\lambda_{j} \in \mathbb{R}_{*}$ such that

$$
\mathbf{u}_{j}+\mathbf{w}_{j}=\lambda_{j}\left(\mathbf{u}_{j}^{\prime}+\mathbf{w}_{j}^{\prime}\right) \text { for } 1 \leq j \leq d .
$$

Thus, multiplying (13) on the left side by $\mathbf{u}_{j}^{T}$ and, by using that $\mathbf{u}_{j}^{T} \mathbf{u}_{j}=1$ and $\mathbf{u}_{j}^{T} \mathbf{w}_{j}=0$, we obtain

$$
\lambda_{j}=\frac{1}{\mathbf{u}_{j}^{T} \mathbf{u}_{j}^{\prime}+\mathbf{u}_{j}^{T} \mathbf{w}_{j}^{\prime}} \text { for } 1 \leq j \leq d .
$$

Hence,

$$
\mathbf{w}_{j}=\frac{\mathbf{u}_{j}^{\prime}+\mathbf{w}_{j}^{\prime}}{\mathbf{u}_{j}^{T} \mathbf{u}_{j}^{\prime}+\mathbf{u}_{j}^{T} \mathbf{w}_{j}^{\prime}}-\mathbf{u}_{j}
$$

defines a $\mathcal{C}^{\infty}$-function from the open set $\mathcal{V}_{j}=\left\{\mathbf{w}_{j} \in \operatorname{span}\left\{\mathbf{u}_{j}^{\prime}\right\}^{\perp}: \mathbf{u}_{j}^{T} \mathbf{u}_{j}^{\prime}+\mathbf{u}_{j}^{T} \mathbf{w}_{j}^{\prime} \neq 0\right\}$ to $\operatorname{span}\left\{\mathbf{u}_{j}\right\}^{\perp}$ for each $1 \leq j \leq d$. Moreover,

$$
1+\left\|\mathbf{w}_{j}\right\|_{j, 2}^{2}=\left\|\frac{\mathbf{u}_{j}^{\prime}+\mathbf{w}_{j}^{\prime}}{\mathbf{u}_{j}^{T} \mathbf{u}_{j}^{\prime}+\mathbf{u}_{j}^{T} \mathbf{w}_{j}^{\prime}}\right\|_{j, 2}^{2}
$$


holds for $1 \leq j \leq d$. Observe that $\mathbf{z}$ can be written as

$$
\mathbf{z}=\beta \bigotimes_{j=1}^{d}\left(\mathbf{u}_{j}+\mathbf{w}_{j}\right)=\left(\beta \prod_{j=1}^{d} \sqrt{1+\left\|\mathbf{w}_{j}\right\|_{j, 2}^{2}}\right) \bigotimes_{j=1}^{d} \mathbf{z}_{j},
$$

where

$$
\mathbf{z}_{j}:=\frac{1}{\sqrt{1+\left\|\mathbf{w}_{j}\right\|_{j, 2}^{2}}} \mathbf{u}_{j}+\frac{1}{\sqrt{1+\left\|\mathbf{w}_{j}\right\|_{j, 2}^{2}}} \mathbf{w}_{j}
$$

has norm one for $1 \leq j \leq d$. In addition,

$$
\mathbf{z}=\left(\beta^{\prime} \prod_{j=1}^{d} \sqrt{1+\left\|\mathbf{w}_{j}^{\prime}\right\|_{j, 2}^{2}}\right) \bigotimes_{j=1}^{d} \mathbf{z}_{j}^{\prime}
$$

where

$$
\mathbf{z}_{j}^{\prime}:=\frac{1}{\sqrt{1+\left\|\mathbf{w}_{j}^{\prime}\right\|_{j, 2}^{2}}} \mathbf{u}_{j}^{\prime}+\frac{1}{\sqrt{1+\left\|\mathbf{w}_{j}^{\prime}\right\|_{j, 2}^{2}}} \mathbf{w}_{j}^{\prime}
$$

has norm one for $1 \leq j \leq d$. Thus,

$$
\beta=\beta^{\prime} \frac{\prod_{j=1}^{d} \sqrt{1+\left\|\mathbf{w}_{j}^{\prime}\right\|_{j, 2}^{2}}}{\prod_{j=1}^{d} \sqrt{1+\left\|\mathbf{w}_{j}\right\|_{j, 2}^{2}}}=\beta^{\prime} \frac{\prod_{j=1}^{d} \sqrt{1+\left\|\mathbf{w}_{j}^{\prime}\right\|_{j, 2}^{2}}}{\prod_{j=1}^{d}\left\|\frac{\mathbf{u}_{j}^{\prime}+\mathbf{w}_{j}^{\prime}}{\| \mathbf{u}_{j}^{T} \mathbf{u}_{j}^{\prime}+\mathbf{u}_{j}^{T} \mathbf{w}_{j}^{\prime}}\right\|_{j, 2}}
$$

clearly defines a $\mathcal{C}^{\infty}$-function from the open set $\mathcal{V}_{1} \times \cdots \times \mathcal{V}_{d} \times \mathbb{R}_{*} \subset \mathcal{U}_{\mathbf{u}^{\prime}}$ to $\mathcal{U}_{\mathbf{u}}$. Finally, we conclude that

$$
\varphi_{\mathbf{u}^{\prime}}\left(\mathfrak{U}(\mathbf{u}) \cap \mathfrak{U}\left(\mathbf{u}^{\prime}\right)\right)=\mathcal{V}_{1} \times \cdots \times \mathcal{V}_{d} \times \mathbb{R}_{*} \subset \mathcal{U}_{\mathbf{u}^{\prime}},
$$

the map

$$
\varphi_{\mathbf{u}} \circ \varphi_{\mathbf{u}^{\prime}}^{-1}: \varphi_{\mathbf{u}^{\prime}}\left(\mathfrak{U}(\mathbf{u}) \cap \mathfrak{U}\left(\mathbf{u}^{\prime}\right)\right) \longrightarrow \varphi_{\mathbf{u}}\left(\mathfrak{U}(\mathbf{u}) \cap \mathfrak{U}\left(\mathbf{u}^{\prime}\right)\right)
$$

is given by

$$
\left(\mathbf{w}_{1}^{\prime}, \ldots, \mathbf{w}_{d}^{\prime}, \beta^{\prime}\right) \mapsto\left(\frac{\mathbf{u}_{1}^{\prime}+\mathbf{w}_{1}^{\prime}}{\mathbf{u}_{1}^{T} \mathbf{u}_{1}^{\prime}+\mathbf{u}_{1}^{T} \mathbf{w}_{1}^{\prime}}-\mathbf{u}_{1}, \ldots, \frac{\mathbf{u}_{d}^{\prime}+\mathbf{w}_{d}^{\prime}}{\mathbf{u}_{d}^{T} \mathbf{u}_{d}^{\prime}+\mathbf{u}_{d}^{T} \mathbf{w}_{d}^{\prime}}-\mathbf{u}_{d}, \beta^{\prime} \frac{\prod_{j=1}^{d} \sqrt{1+\left\|\mathbf{w}_{j}^{\prime}\right\|_{j, 2}^{2}}}{\prod_{j=1}^{d} \| \frac{\mathbf{u}_{j}^{\prime}+\mathbf{w}_{j}^{\prime}}{\mathbf{u}_{j}^{T} \mathbf{u}_{j}^{\prime}+\mathbf{u}_{j}^{T} \mathbf{w}_{j}^{\prime} \|_{j, 2}}},\right)
$$

and it is $\mathcal{C}^{\infty}$. This follows AT2, AT3 and concludes the proof of the theorem.

The construction of $\mathcal{M}_{N_{1} \cdots N_{d}}$ as an algebraic variety is well-known (see, for example, [15]). More recently, in [16], a structure of smooth manifold is given, in the framework of Banach spaces, for the set of tensors of fixed rank-one. Following [16], it can be shown that the manifold $\mathcal{M}_{N_{1} \cdots N_{d}}$ is also a principal bundle as follows. Consider the Grassmann manifold of one-dimensional, subspaces of $\mathbb{R}^{N_{j}}$, denoted by $\mathbb{G}_{1}\left(\mathbb{R}^{N_{j}}\right)$, for $1 \leq j \leq d$ and define the surjective map

$$
\pi: \mathcal{M}_{N_{1} \cdots N_{d}} \longrightarrow \mathbb{G}_{1}\left(\mathbb{R}^{N_{1}}\right) \times \cdots \times \mathbb{G}_{1}\left(\mathbb{R}^{N_{d}}\right), \quad \mathbf{u}=\lambda \bigotimes_{j=1}^{d} \mathbf{u}_{j} \mapsto\left(\operatorname{span}\left\{\mathbf{u}_{j}\right\}\right)_{j=1}^{d}
$$

Then, for each $\mathbf{u}=\lambda \bigotimes_{j=1}^{d} \mathbf{u}_{j} \in \mathcal{M}_{N_{1} \cdots N_{d}}$, it holds that

$$
\pi^{-1}(\pi(\mathbf{u}))=\bigotimes_{j=1}^{d} \operatorname{span}\left\{\mathbf{u}_{j}\right\} \backslash\{\mathbf{0}\}=\operatorname{span}\{\mathbf{u}\} \backslash\{\mathbf{0}\} \cong \mathbb{R}_{*}
$$


Consequently, $\mathcal{M}_{N_{1} \cdots N_{d}}$ is also a principal bundle with base space $\mathbb{G}_{1}\left(\mathbb{R}^{N_{1}}\right) \times \cdots \times$ $\mathbb{G}_{1}\left(\mathbb{R}^{N_{d}}\right)$ and fibre $\mathbb{R}_{*}$. It allows for decomposing the tangent space at $\mathbf{u} \in \mathcal{M}_{N_{1} \cdots N_{d^{\prime}}}$ denoted $\mathrm{T}_{\mathbf{u}} \mathcal{M}_{N_{1} \cdots N_{d}}$ into the vertical and horizontal spaces:

$$
\mathrm{T}_{\mathbf{u}} \mathcal{M}_{N_{1} \cdots N_{d}}=X_{\mathbf{u}}=\mathrm{H}_{\mathbf{u}} \mathcal{M}_{N_{1} \cdots N_{d}}+\mathrm{V}_{\mathbf{u}} \mathcal{M}_{N_{1} \cdots N_{d}},
$$

where

$$
\mathrm{H}_{\mathbf{u}} \mathcal{M}_{N_{1} \cdots N_{d}}:=\operatorname{span}\left\{\mathbf{u}_{1}\right\}^{\perp} \times \cdots \times \operatorname{span}\left\{\mathbf{u}_{d}\right\}^{\perp}
$$

and $\mathrm{V}_{\mathbf{u}} \mathcal{M}_{N_{1} \cdots N_{d}}:=\mathbb{R}$

\section{On the First Order Optimality Conditions for the PGD}

The goal of this section is given $A \in \operatorname{GL}\left(N_{1} N_{2} \cdots N_{d}\right)$ and $\mathbf{f} \in \mathbb{R}^{N_{1} N_{2} \cdots N_{d}}$ characterize the points in the manifold $\mathcal{M}_{N_{1} \cdots N_{d}}$ satisfying the first order optimality conditions of the problem

$$
\min _{\mathbf{z} \in \mathcal{M}_{N_{1} \cdots N_{d}}} J_{A, \mathbf{f}}(\mathbf{z})
$$

Recall that the map $J_{A, \mathbf{f}}$ is defined in the whole ambient space $\mathbb{R}^{N_{1}} \otimes \cdots \otimes \mathbb{R}^{N_{d}}$. We will denote its derivative at $\mathbf{u} \in \mathbb{R}^{N_{1}} \otimes \cdots \otimes \mathbb{R}^{N_{d}}$ by $J_{A, \mathbf{f}}^{\prime}(\mathbf{u})=(\mathbf{f}-A \mathbf{u})^{T}$, which is a bounded linear map from $\mathbb{R}^{N_{1}} \otimes \cdots \otimes \mathbb{R}^{N_{d}}$ to $\mathbb{R}$. From Theorem 3, we known that $\mathcal{M}_{N_{1} \cdots N_{d}}$ is a $\mathcal{C}^{\infty}$ manifold and hence it allows us to write the constrained map $\left.J_{A, \mathbf{f}}\right|_{\mathcal{M}_{N_{1} \cdots N_{d}}}$ as follows. Since

$$
J_{A, \mathbf{f}}: \mathbb{R}^{N_{1}} \otimes \cdots \otimes \mathbb{R}^{N_{d}} \longrightarrow \mathbb{R},
$$

and $\mathcal{M}_{N_{1} \cdots N_{d}} \subset \mathbb{R}^{N_{1}} \otimes \cdots \otimes \mathbb{R}^{N_{d}}$, we can take into account the standard inclusion map

$$
i: \mathcal{M}_{N_{1} \cdots N_{d}} \longrightarrow \mathbb{R}^{N_{1}} \otimes \cdots \otimes \mathbb{R}^{N_{d}}, \quad \mathbf{z} \mapsto \mathbf{z}
$$

in order to write $\left.J_{A, \mathbf{f}}\right|_{\mathcal{M}_{N_{1} \cdots N_{d}}}=\left(J_{A, \mathbf{f}} \circ i\right)$.

Definition 4. We say that $\mathbf{u} \in \mathcal{M}_{N_{1} \cdots N_{d}}$ is a critical point for $J_{A, \mathbf{f}}$ in $\mathcal{M}_{N_{1} \cdots N_{d}}$ if

$$
T_{\mathbf{u}} J_{A, \mathbf{f}}(\mathbf{v}):=\left[D\left(J_{A, \mathbf{f}} \circ \varphi_{\mathbf{u}}^{-1}\right)\left(\varphi_{\mathbf{u}}(\mathbf{u})\right)\right](\mathbf{v})=0
$$

holds for all $\mathbf{v} \in \mathrm{T}_{\mathbf{u}} \mathcal{M}_{N_{1} \cdots N_{d}}$. Clearly, if $\mathbf{u}$ is an extremal point for $J_{A, \mathbf{f}}$ in $\mathcal{M}_{N_{1} \cdots N_{d^{\prime}}}$ then it is also a critical point for $J_{A, \mathrm{f}}$ in $\mathcal{M}_{N_{1} \cdots N_{d}}$.

Observe that we can write

$$
J_{A, \mathbf{f}} \circ \varphi_{\mathbf{u}}^{-1}=J_{A, \mathbf{f}} \circ\left(i \circ \varphi_{\mathbf{u}}^{-1}\right),
$$

where on the left side of the equality, we consider $J_{A, \mathrm{f}}$ over $\mathcal{M}_{N_{1} \cdots N_{d}}$, whereas, in the right one, $J_{A, \mathrm{f}}$ is considered defined over the whole space. Thus, by using the chain rule, we have

$$
\begin{aligned}
D\left(J_{A, \mathbf{f}} \circ \varphi_{\mathbf{u}}^{-1}\right)\left(\varphi_{\mathbf{u}}(\mathbf{u})\right) & =D\left(J_{A, \mathbf{f}} \circ\left(i \circ \varphi_{\mathbf{u}}^{-1}\right)\right)\left(\varphi_{\mathbf{u}}(\mathbf{u})\right) \\
& =J_{A, \mathbf{f}}^{\prime}(\mathbf{u}) \circ D\left(i \circ \varphi_{\mathbf{u}}^{-1}\right)\left(\varphi_{\mathbf{u}}(\mathbf{u})\right),
\end{aligned}
$$

that is,

$$
T_{\mathbf{u}} J_{A, \mathbf{f}}=J_{A, \mathbf{f}}^{\prime}(\mathbf{u}) \circ T_{\mathbf{u}} i=(\mathbf{f}-A \mathbf{u})^{T} T_{\mathbf{u}} i .
$$


In order to compute (15), we consider first the standard inclusion map $i$ that in local coordinates in a neighbourhood of $\mathbf{u}=\lambda \bigotimes_{j=1}^{d} \mathbf{u}_{j} \in \mathcal{M}_{N_{1} \cdots N_{d}}$ looks like

$$
\left(i \circ \varphi_{\mathbf{u}}^{-1}\right)\left(\mathbf{w}_{1}, \ldots, \mathbf{w}_{d}, \beta\right)=\beta \bigotimes_{j=1}^{w}\left(\mathbf{u}_{j}+\mathbf{w}_{j}\right) .
$$

Here, $\left(i \circ \varphi_{\mathbf{u}}^{-1}\right): \mathcal{U}_{\mathbf{u}} \longrightarrow \mathbb{R}^{N_{1}} \otimes \cdots \otimes \mathbb{R}^{N_{d}}$. Hence, its derivative as a morphism (a map between manifolds)

$$
\mathrm{T}_{\mathbf{u}} i=D\left(i \circ \varphi_{\mathbf{u}}^{-1}\right)(\mathbf{0}, \ldots, \mathbf{0}, \lambda): \mathrm{T}_{\mathbf{u}} \mathcal{M}_{N_{1} \cdots N_{d}} \longrightarrow \mathbb{R}^{N_{1}} \otimes \cdots \otimes \mathbb{R}^{N_{d}},
$$

is given by

$$
\mathrm{T}_{\mathbf{u}} i\left(\beta, \mathbf{w}_{1}, \ldots, \mathbf{w}_{d}\right)=\sum_{j=1}^{d} \mathbf{w}_{j} \otimes \mathbf{u}_{[j]}+\frac{\beta}{\lambda} \mathbf{u}
$$

where

$$
\mathbf{w}_{j} \otimes \mathbf{u}_{[j]}:=\lambda \mathbf{u}_{1} \otimes \cdots \otimes \mathbf{u}_{j-1} \otimes \mathbf{w}_{j} \otimes \mathbf{u}_{j+1} \otimes \cdots \otimes \mathbf{u}_{d} .
$$

for $1 \leq j \leq d$. From (15), we have that $\mathbf{u}^{*}=\lambda^{*} \bigotimes_{j=1}^{d} \mathbf{u}_{i}^{*}$ is a critical point for $J_{A, \mathbf{f}}$ in $\mathcal{M}_{N_{1} \cdots N_{d}}$ if and only if

$$
\left(\mathbf{f}-A \mathbf{u}^{*}\right)^{T} T_{\mathbf{u}^{*}} i\left(\mathbf{w}_{1}, \ldots, \mathbf{w}_{d}, \beta\right)=\mathbf{0}, \text { holds for all }\left(\mathbf{w}_{1}, \ldots, \mathbf{w}_{d}, \beta\right) \in \mathrm{T}_{\mathbf{u}^{*}} \mathcal{M}_{N_{1} \cdots N_{d^{\prime}}}
$$

that is, it is equivalent to state that

$$
\left(\mathbf{f}-A \mathbf{u}^{*}\right)^{T}\left(\sum_{j=1}^{d} \mathbf{w}_{j} \otimes \mathbf{u}_{[j]}^{*}+\frac{\beta}{\lambda} \mathbf{u}^{*}\right)=\mathbf{0} \text {, holds for all }\left(\mathbf{w}_{1}, \ldots, \mathbf{w}_{d}, \beta\right) \in \mathrm{T}_{\mathbf{u}^{*}} \mathcal{M}_{N_{1} \cdots N_{d}} .
$$

Now, we will prove the following result that characterizes the set of critical points for $J_{A, \mathrm{f}}$ in $\mathcal{M}_{N_{1} \cdots N_{d}}$ as the stationary points of a vector field in $\mathbb{R}^{N_{1}} \times \cdots \times \mathbb{R}^{N_{d}}$, and it is the main result of this paper.

Theorem 4. Given $A \in \operatorname{GL}\left(N_{1} N_{2} \cdots N_{d}\right)$ and $\mathbf{f} \in \mathbb{R}^{N_{1} N_{2} \cdots N_{d}}$. Then, $\mathbf{u}^{*}=\bigotimes_{j=1}^{d} \mathbf{u}_{i}^{*} \in$ $\mathcal{M}_{N_{1} \cdots N_{d}}$ is a critical point for $J_{A, \mathbf{f}}$ in $\mathcal{M}_{N_{1} \cdots N_{d}}$ if and only if $\mathbf{X}_{A, \mathbf{f}}\left(\mathbf{u}_{1}^{*}, \ldots, \mathbf{u}_{d}^{*}\right)=\mathbf{0}$, where $\mathbf{X}_{A, \mathbf{f}}$ is a vector field in $\mathcal{M}_{N_{1} \cdots N_{d}}$ given by

$$
\mathbf{X}_{A, \mathbf{f}}\left(\mathbf{u}_{1}, \ldots, \mathbf{u}_{d}\right)=\left(\mathbf{y}_{1}, \ldots, \mathbf{y}_{d}\right)
$$

Here,

$$
\mathbf{y}_{j}:=\left(\mathbf{u}_{1} \otimes \cdots \otimes \mathbf{u}_{j-1} \otimes i d_{\mathbb{R}^{N_{j}}} \otimes \mathbf{u}_{j+1} \otimes \cdots \otimes \mathbf{u}_{d}\right)^{T}\left(\mathbf{f}-A\left(\mathbf{u}_{1} \otimes \cdots \otimes \mathbf{u}_{d}\right)\right)
$$

for $1 \leq j \leq d$.

Proof. Since $\mathbf{u}^{*}=\bigotimes_{j=1}^{d} \mathbf{u}_{i}^{*} \in \mathcal{M}_{N_{1} \cdots N_{d}}$ is a critical point for $J_{A, \mathrm{f}}$ in $\mathcal{M}_{N_{1} \cdots N_{d}}$ if and only if (17) holds for $\left(\mathbf{w}_{1}, \ldots, \mathbf{w}_{d}, \beta\right) \in \mathrm{T}_{\mathbf{u}^{*}} \mathcal{M}_{N_{1} \cdots N_{d}}$. Then, (17) is equivalent to prove that

$$
\left(\mathbf{f}-A \mathbf{u}^{*}\right)^{T} \mathbf{u}^{*}=\mathbf{0},
$$

and

$$
\left(\mathbf{f}-A \mathbf{u}^{*}\right)^{T}\left(\mathbf{w}_{j} \otimes \mathbf{u}_{[j]}^{*}\right)=\mathbf{0} \text { holds for all } \mathbf{w}_{j} \in \operatorname{span}\left\{\mathbf{u}_{j}\right\}^{\perp} \text { and } 1 \leq j \leq d .
$$


Since $\mathbf{w}_{j} \otimes \mathbf{u}_{[j]}^{*}=\mathbf{u}^{*}$ when $\mathbf{w}_{j}=\mathbf{u}_{j}^{*}$ for $1 \leq j \leq d$, we conclude that $\mathbf{u}^{*}=\bigotimes_{j=1}^{d} \mathbf{u}_{i}^{*} \in$ $\mathcal{M}_{N_{1} \cdots N_{d}}$ is a critical point for $J_{A, \mathbf{f}}$ in $\mathcal{M}_{N_{1} \cdots N_{d}}$ if and only if

$$
\left(\mathbf{f}-A \mathbf{u}^{*}\right)^{T}\left(\mathbf{w}_{j} \otimes \mathbf{u}_{[j]}^{*}\right)=\mathbf{0} \text { holds for all } \mathbf{w}_{j} \in \mathbb{R}^{N_{j}} \text { and } 1 \leq j \leq d .
$$

Writing the equality in (20) as

$$
\left(\mathbf{f}-A \mathbf{u}_{1}^{*} \otimes \cdots \otimes \mathbf{u}_{d}^{*}\right)^{T}\left(\mathbf{u}_{1}^{*} \otimes \cdots \otimes \mathbf{u}_{j-1}^{*} \otimes i d_{\mathbb{R}^{N_{j}}} \otimes \mathbf{u}_{j+1}^{*} \otimes \cdots \otimes \mathbf{u}_{d}^{*}\right) \mathbf{w}_{j}=\mathbf{0},
$$

we conclude that it is equivalent to state that

$$
\left(\mathbf{f}-A \mathbf{u}_{1}^{*} \otimes \cdots \otimes \mathbf{u}_{d}^{*}\right)^{T}\left(\mathbf{u}_{1}^{*} \otimes \cdots \otimes \mathbf{u}_{j-1}^{*} \otimes i d_{\mathbb{R}^{N_{j}}} \otimes \mathbf{u}_{j+1}^{*} \otimes \cdots \otimes \mathbf{u}_{d}^{*}\right)=\mathbf{0}^{T}
$$

for $1 \leq j \leq d$. This concludes the proof of theorem

Remark 1. From (17), we can deduce that $\mathbf{u}^{*}=\bigotimes_{j=1}^{d} \mathbf{u}_{i}^{*}$ is a critical point for $J_{A, \mathbf{f}}$ in $\mathcal{M}_{N_{1} \cdots N_{d}}$ if and only if

$$
\left(\mathbf{f}-A \mathbf{u}^{*}\right)^{T}\left(\sum_{j=1}^{d} \mathbf{w}_{j} \otimes \mathbf{u}_{[j]}^{*}\right)=\mathbf{0} \text { holds for all }\left(\mathbf{w}_{1}, \ldots, \mathbf{w}_{d}\right) \in \mathbb{R}^{N_{1}} \times \cdots \times \mathbb{R}^{N_{d}} .
$$

Statement (21) was first introduced in [2,3] as a step to enrich the approximation basis in the PGD algorithm. From the proof of Theorem 4, we have that (21) is also equivalent to (18) and (19).

There are several strategies in the literature that can be used to solve $\mathbf{X}_{A, \mathbf{f}}(\mathbf{u})=\mathbf{0}$. The first one, closely related to the one used in $[2,3]$, is to find a fixed point of the map

$$
F_{A, \mathbf{f}}\left(\mathbf{u}_{1}, \ldots, \mathbf{u}_{d}\right):=\mathbf{X}_{A, \mathbf{f}}\left(\mathbf{u}_{1}, \ldots, \mathbf{u}_{d}\right)-\left(\mathbf{u}_{1}, \ldots, \mathbf{u}_{d}\right)
$$

One of the most popular numerical strategies to compute an approximated value of $\mathbf{u}^{*}$ such that $\mathbf{X}_{A, \mathbf{f}}\left(\mathbf{u}^{*}\right)=\mathbf{0}$ is founded under the following argument. Since the $\mathbf{y}_{1}$ is equal to

$$
\begin{array}{r}
\left(i d_{\mathbb{R}^{N_{1}}} \otimes \mathbf{u}_{2} \otimes \cdots \otimes \mathbf{u}_{d}\right)^{T}\left(\mathbf{f}-A \mathbf{u}_{1} \otimes \cdots \otimes \mathbf{u}_{d}\right)= \\
\left(i d_{\mathbb{R}^{N_{1}}} \otimes \mathbf{u}_{2} \otimes \cdots \otimes \mathbf{u}_{d}\right)^{T} \mathbf{f}-\left(i d_{\mathbb{R}^{N_{1}}} \otimes \mathbf{u}_{2} \otimes \cdots \otimes \mathbf{u}_{d}\right)^{T} A\left(\mathbf{u}_{1} \otimes \cdots \otimes \mathbf{u}_{d}\right)= \\
\left(i d_{\mathbb{R}^{N_{1}}} \otimes \mathbf{u}_{2} \otimes \cdots \otimes \mathbf{u}_{d}\right)^{T} \mathbf{f}-\left(i d_{\mathbb{R}^{N_{1}}} \otimes \mathbf{u}_{2} \otimes \cdots \otimes \mathbf{u}_{d}\right)^{T} A\left(i d_{\mathbb{R}^{N_{1}}} \otimes \mathbf{u}_{2} \cdots \otimes \mathbf{u}_{d}\right) \mathbf{u}_{1},
\end{array}
$$

we can choose randomly some vectors $\mathbf{u}_{j} \in \mathbb{R}^{N_{j}}$ for $2 \leq j \leq d$ and then try to solve the linear system

$$
\left(i d_{\mathbb{R}^{N_{1}}} \otimes \mathbf{u}_{2} \otimes \cdots \otimes \mathbf{u}_{d}\right)^{T} A\left(i d_{\mathbb{R}^{N_{1}}} \otimes \mathbf{u}_{2} \cdots \otimes \mathbf{u}_{d}\right) \mathbf{u}_{1}=\left(i d_{\mathbb{R}^{N_{1}}} \otimes \mathbf{u}_{2} \otimes \cdots \otimes \mathbf{u}_{d}\right)^{T} \mathbf{f} .
$$

By using Least Squares, we can compute a $\mathbf{u}_{1}^{\prime}$, and next we can proceed in a similar way iteratively with each of the other components $\mathbf{y}_{2}, \ldots, \mathbf{y}_{d}$ as follows. After the step $i \geq 1$, we know that $\mathbf{u}_{1}^{\prime}, \mathbf{u}_{2}^{\prime}, \ldots, \mathbf{u}_{i}^{\prime}$. Then, by choosing randomly $\mathbf{u}_{i+1}, \ldots \mathbf{u}_{d}$, we can solve, by using Least Squares, the linear system

$$
\begin{array}{r}
\left(\mathbf{u}_{1}^{\prime} \otimes \cdots \otimes \mathbf{u}_{i}^{\prime} \otimes i d_{\mathbb{R}^{N_{i}}} \otimes \mathbf{u}_{i+1} \otimes \cdots \otimes \mathbf{u}_{d}\right)^{T} A\left(i d_{\mathbb{R}^{N_{1}}} \otimes \mathbf{u}_{2} \cdots \otimes \mathbf{u}_{d}\right) \mathbf{u}_{i} \\
=\left(\mathbf{u}_{1}^{\prime} \otimes \cdots \otimes \mathbf{u}_{i}^{\prime} \otimes i d_{\mathbb{R}^{N_{i}}} \otimes \mathbf{u}_{i+1} \otimes \cdots \otimes \mathbf{u}_{d}\right)^{T} \mathbf{f},
\end{array}
$$

to compute $\mathbf{u}_{i}^{\prime}$. We can proceed cyclically until $\left\|\mathbf{f}-A\left(\mathbf{u}_{1}^{\prime} \otimes \cdots \otimes \mathbf{u}_{d}^{\prime}\right)\right\|<\varepsilon$ holds. This strategy is known by the name of the Alternating Least Squares (ALS).

From (17), we have that $\mathbf{u}^{*}$ is a critical point of $J_{A, \mathbf{f}}$ in $\mathcal{M}_{N_{1} \cdots N_{d}}$ if and only if

$$
\left(\mathbf{f}-A \mathbf{u}^{*}\right) \perp \mathrm{T}_{\mathbf{u}^{*}} i\left(\mathrm{~T}_{\mathbf{u}^{*}} \mathcal{M}_{N_{1} \cdots N_{d}}\right)
$$


holds, that is, the residual at $\mathbf{u}^{*}$ is orthogonal to linear subspace $\mathrm{T}_{\mathbf{u}^{*}} i\left(\mathrm{~T}_{\mathbf{u}^{*}} \mathcal{M}_{N_{1} \cdots N_{d}}\right)$. Observe that $\mathbf{w}_{j} \otimes \mathbf{u}_{[j]} \in V_{j}(\mathbf{u})$, where

$$
V_{j}(\mathbf{u}):=\operatorname{span}\left\{\mathbf{u}_{1}\right\} \otimes \cdots \otimes \operatorname{span}\left\{\mathbf{u}_{j-1}\right\} \otimes \operatorname{span}\left\{\mathbf{u}_{j}\right\}^{\perp} \otimes \operatorname{span}\left\{\mathbf{u}_{j+1}\right\} \otimes \cdots \otimes \operatorname{span}\left\{\mathbf{u}_{d}\right\}
$$

is an $N_{j}$-1-dimensional subspace of $\mathbb{R}^{N_{1}} \otimes \cdots \otimes \mathbb{R}^{N_{d}}$ for $1 \leq j \leq d$. Then, the next result explicitly describes the linear subspace $\mathrm{T}_{\mathbf{u}^{*}} i\left(\mathrm{~T}_{\mathbf{u}^{*}} \mathcal{M}_{N_{1} \cdots N_{d}}\right)$.

Proposition 1. The linear map $T_{\mathbf{u}} i: \mathrm{T}_{\mathbf{u}} \mathcal{M}_{N_{1} \cdots N_{d}} \longrightarrow \mathbb{R}^{N_{1}} \otimes \cdots \otimes \mathbb{R}^{N_{d}}$ is injective. Moreover,

$$
\mathrm{T}_{\mathbf{u}} i\left(\mathrm{~T}_{\mathbf{u}} \mathcal{M}_{N_{1} \cdots N_{d}}\right)=\bigoplus_{j=1}^{d} V_{j}(\mathbf{u}) \oplus \operatorname{span}\{\mathbf{u}\} \cong \mathrm{T}_{\mathbf{u}} \mathcal{M}_{N_{1} \cdots N_{d}} .
$$

Proof. Assume that

$$
\mathrm{T}_{\mathbf{u}} i\left(\beta, \mathbf{w}_{1}, \ldots, \mathbf{w}_{d}\right)=\sum_{j=1}^{d} \mathbf{w}_{j} \otimes \mathbf{u}_{[j]}+\frac{\beta}{\lambda} \mathbf{u}=\mathbf{0} .
$$

Since $\mathbf{u} \perp\left(\mathbf{w}_{j} \otimes \mathbf{u}_{[j]}\right)$ for $1 \leq j \leq d$ and $\left(\mathbf{w}_{i} \otimes \mathbf{u}_{[j]}\right) \perp\left(\mathbf{w}_{j} \otimes \mathbf{u}_{[j]}\right)$ for all $i \neq j$, then

$$
\beta=0 \text { and } \mathbf{w}_{i} \otimes \mathbf{u}_{[j]}=\mathbf{0} \text { for } 1 \leq j \leq d .
$$

Since $\mathbf{u}_{[j]} \neq \mathbf{0}$, then $\mathbf{w}_{j}=\mathbf{0}$ for $1 \leq j \leq d$. This follows the first statement. To prove the second one, we remark that the inclusion

$$
\mathrm{T}_{\mathbf{u}} i\left(\mathrm{~T}_{\mathbf{u}} \mathcal{M}_{N_{1} \cdots N_{d}}\right) \subset \bigoplus_{j=1}^{d} V_{j}(\mathbf{u}) \oplus \operatorname{span}\{\mathbf{u}\}
$$

between both subspaces is trivial. Clearly,

$$
\operatorname{dim} \mathrm{T}_{\mathbf{u}} i\left(\mathrm{~T}_{\mathbf{u}} \mathcal{M}_{N_{1} \cdots N_{d}}\right)=\operatorname{dim}\left(\bigoplus_{j=1}^{d} V_{j}(\mathbf{u}) \oplus \operatorname{span}\{\mathbf{u}\}\right)
$$

holds and then the second statement is also proved.

\section{Conclusions}

In this paper, a geometric upgrade of the PGD algorithm is proposed. To this end, we endow a set of tensors of fixed rank-one with a smooth manifold structure. This construction provides the solutions of a non-convex optimization problem by using a set of stationary points of a vector field. This new perspective allows for the characterization of the behaviour of the solutions of the Greedy Rank One Algorithm which could not be achieved with the ALS strategy used in [8].

Author Contributions: Investigation, writing and revision: A.F., L.H., N.M., M.C.M. and E.N. All authors have read and agreed to the published version of the manuscript.

Funding: This research was funded by the GVA/2019/124 grant from Generalitat Valenciana and by the RTI2018-093521-B-C32 grant from the Ministerio de Ciencia, Innovación y Universidades.

Conflicts of Interest: The authors declare no conflict of interest. The funders had no role in the design of the study; in the collection, analyses, or interpretation of data; in the writing of the manuscript, or in the decision to publish the results. 


\section{References}

1. Falcó, A.; Montés, N.; Chinesta, F.; Hilario, L.; Mora, M.C. On the existence of a Progressive Variational Vademecum based on the Proper Generalized Decomposition for a Class of Elliptic Parametrized Problems. J. Comput. Appl. Math. 2018, 330, $1093-1107$. [CrossRef]

2. Ammar, A.; Mokdad, B.; Chinesta, F.; Keunings, R. A new family of solvers for some classes of multidimensional partial differential equations encountered in Kinetic Theory modelling Complex Fluids. J. Non-Newton. Fluid Mech. 2006, 139, 153-176. [CrossRef]

3. Ammar, A.; Mokdad, B.; Chinesta, F.; Keunings, R. A new family of solvers for some classes of multidimensional partial differential equations encountered in Kinetic Theory modelling Complex Fluids. Part II: Transient Simulations using Space-Time Separated Representations. J. Non-Newton. Fluid Mech. 2007, 144, 98-121. [CrossRef]

4. Falcó, A.; Nouy, A. A Proper Generalized Decomposition for the solution of elliptic problems in abstract form by using a functional Eckart- Young approach. J. Math. Anal. Appl. 2011, 376, 469-480. [CrossRef]

5. Chinesta, F.; Leygue, A.; Bordeu, F.; Aguado, J.V.; Cueto, E.; González, D.; Alfaro, I.; Ammar, A.; Huerta, A. PGD-Based computational vademecum for efficient Design, Optimization and Control. Arch. Comput. Methods Eng. 2013, $20,31-49$. [CrossRef]

6. Chinesta, F.; Ladevéze, P. Separated Representations and PGD-Based Model Reduction; Springer International Centre for Mechanical Sciences: Cham, Switzerland, 2014.

7. Falcó, A.; Nouy, A. Proper Generalized Decomposition for Nonlinear Convex Problems in Tensor Banach Spaces. Numer. Math. 2012, 121, 503-530. [CrossRef]

8. Ammar, A.; Chinesta, F.; Falcó, A. On the convergence of a greedy rank-one update algorithm for a class of linear systems. Arch. Comput. Methods Eng. 2010, 17, 473-486. [CrossRef]

9. El Hamidi, A.; Osman, H.; Jazar, M. On the convergence of alternating minimization methods in variational PGD. Comput. Optim. Appl. 2017, 68, 455-472. [CrossRef]

10. Graham, A. Kronecker Products and Matrix Calculus with Applications; John Wiley: Hoboken, NJ, USA, 1981.

11. Van Loan, C.F. The ubiquitous Kronecker product, J.Comput. Appl. Math. 2000, 123, 85-100. [CrossRef]

12. Beylkin, G.; Mohlenkamp, M.J. Algorithms for Numerical Analysis in High Dimensions. SIAM J. Sci. Comput. 2005, 26, 2133-2159. [CrossRef]

13. de Silva, V.; Lim, L.-H. Tensor Rank and Ill-posedness of the best low-rank approximation problem. SIAM J. Matrix Anal. Appl. 2008, 30, 1084-1127. [CrossRef]

14. Lang, S. Differential and Riemannian Manifolds; Graduate Texts in Mathematics; Springer: Berlin/Heidelberg, Germany, 1995; Volume 160.

15. Landsberg, J.M. Tensor: Geometry and Applications. Graduate Studies in Mathematics; American Mathematical Society: Providence, Rhode Island, 2012; Volume 128.

16. Falcó, A.; Hackbusch, W.; Nouy, A. On the Dirac-Frenkel Variational Principal on Tensor Banach Spaces. Found. Comput. Math. 2019, 19, 159-204. [CrossRef] 